\title{
Efficient Synthesis of Taurine and Structurally Diverse Substituted Taurines from Aziridines
}

\author{
Libo Hu, Hui Zhu, Da-Ming Du, and Jiaxi Xu*
}

\begin{abstract}
Beijing National Laboratory for Molecular Sciences (BNLMS), Key Laboratory of Bioorganic Chemistry and Molecular Engineering of Ministry of Education, College of Chemistry and Molecular Engineering, Peking University, Beijing 100871, People's Republic of China jxxu@pku.edu.cn
\end{abstract}

\section{Supplemental Materials}

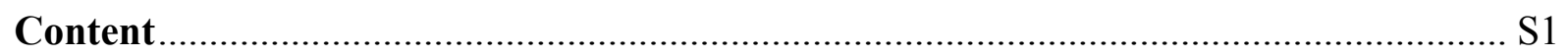

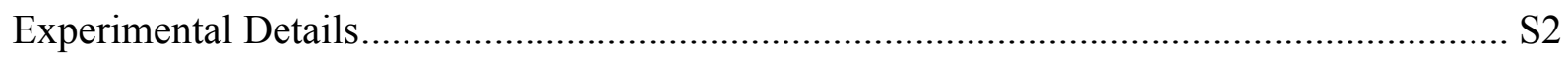

Analytical Data of Taurine and Known Substituted Taurines .............................................. S2

Copies of ${ }^{1} \mathrm{H}$ and ${ }^{13} \mathrm{C}$ NMR Spectra of the Acetamides and N-Acetyl Substituted Taurines S4

Copies of ${ }^{13} \mathrm{C}$ NMR Spectra of the Known Substituted Taurines and ${ }^{1} \mathrm{H}$ and ${ }^{13} \mathrm{C}$ NMR Spectra of Taurine and the Unknown Substituted Taurines S8 


\section{Experimental Details}

General. Aziridine and substituted aziridines were prepared from the corresponding amino alcohols via the Wenker reaction according to literature. ${ }^{1}$ Their analytic data are identical to the reported ones. ${ }^{1-8}$ Other reactants are commercial available and were used without further purification. All solvents were used without purification. ${ }^{1} \mathrm{H}$ NMR spectra were recorded at 200 or $300 \mathrm{MHz}$ in $\mathrm{D}_{2} \mathrm{O}$ and ${ }^{13} \mathrm{C}$ NMR spectra were recorded at 50 or $75.5 \mathrm{MHz}$ in $\mathrm{HCO}_{2} \mathrm{H}$ with $\mathrm{HCO}_{2} \mathrm{H}$ as an internal standard at $166.3 \mathrm{ppm}$. IR spectra were determined in $\mathrm{KBr}$. MS spectra were obtained on an ESI mass spectrometer. Elemental analyses were carried our on an elemental analyzer. Optical rotations were measured on a polarimeter with a thermally jacketed $10 \mathrm{~cm}$ cell (concentration $c$ expressed as $\mathrm{g} / 100 \mathrm{~mL}$ ).

\section{Analytical Data of Taurine and Known Substituted Taurines}

\section{(S)-2-Amino-3-phenylpropane-1-sulfonic acid (4a)}

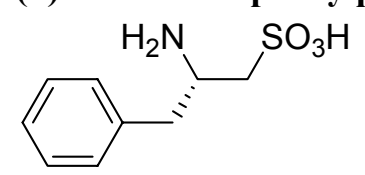

Colorless crystals, m.p. $340-3{ }^{\circ} \mathrm{C}(\mathrm{dec})$; Lit. ${ }^{1}$ m.p. $>330{ }^{\circ} \mathrm{C} .[\alpha]^{20}{ }_{\mathrm{D}}=-2.0\left(c\right.$ 1.13, $\left.\mathrm{H}_{2} \mathrm{O}\right)$; Lit. ${ }^{1}$ $[\alpha]^{20}{ }_{\mathrm{D}}=-3.6\left(c 1, \mathrm{H}_{2} \mathrm{O}\right) .{ }^{13} \mathrm{C} \mathrm{NMR}\left(75.5 \mathrm{MHz}, \mathrm{HCO}_{2} \mathrm{H}\right) \delta: 38.0,50.8,51.4,127.8,129.1,129.4$, 134.1.

\section{2-Aminoethanesulfonic acid (4c)}<smiles>NCCS(=O)(=O)O</smiles>

Colorless crystals, m.p. $325{ }^{\circ} \mathrm{C}(\mathrm{dec})$; Lit. ${ }^{9}$ m.p. $325-30{ }^{\circ} \mathrm{C} .{ }^{1} \mathrm{H}$ NMR $\left(200 \mathrm{MHz}, \mathrm{D}_{2} \mathrm{O}\right) \delta: 3.13$ (t, $J=6.3 \mathrm{~Hz}, 2 \mathrm{H}), 3.30(\mathrm{t}, J=6.3 \mathrm{~Hz}, 2 \mathrm{H}) ;{ }^{13} \mathrm{C} \mathrm{NMR}\left(50 \mathrm{MHz}, \mathrm{HCO}_{2} \mathrm{H}\right) \delta: 38.5,47.4$.

\section{(S)-2-Amino-3-methylbutane-1-sulfonic acid (4d)}

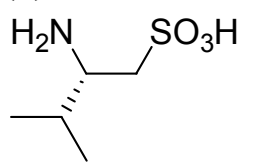

Colorless crystals, m.p. $326-9{ }^{\circ} \mathrm{C}(\mathrm{dec})$; Lit. ${ }^{10}$ m.p. $325-6^{\circ} \mathrm{C} .[\alpha]^{20}{ }_{\mathrm{D}}=+28.3\left(c\right.$ 1.04, $\left.\mathrm{H}_{2} \mathrm{O}\right)$; Lit. ${ }^{1}$ $[\alpha]^{20}{ }_{\mathrm{D}}=+29.6\left(c 0.97, \mathrm{H}_{2} \mathrm{O}\right) .{ }^{13} \mathrm{C} \mathrm{NMR}\left(75.5 \mathrm{MHz}, \mathrm{HCO}_{2} \mathrm{H}\right) \delta: 16.2,17.1,30.3,49.3,54.6$.

\section{2-Amino-2-methylpropane-1-sulfonic acid (4e)}

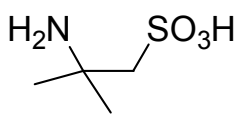

Colorless crystals, m.p. $325{ }^{\circ} \mathrm{C}(\mathrm{dec})$; Lit. ${ }^{11}$ m.p. $>320{ }^{\circ} \mathrm{C} .{ }^{13} \mathrm{C}$ NMR $\left(75.5 \mathrm{MHz}, \mathrm{HCO}_{2} \mathrm{H}\right) \delta$ : 25.3, 54.2, 57.6. 
trans-2-Aminocyclopentane-1-sulfonic acid (4g)<smiles>N[C@@H]1CCC[C@H]1S(=O)(=O)O</smiles>

$( \pm)$

Colorless crystals, m.p. $330{ }^{\circ} \mathrm{C}(\mathrm{dec})$; Lit. ${ }^{12}$ m.p. $330{ }^{\circ} \mathrm{C} .{ }^{13} \mathrm{C}$ NMR $\left(75.5 \mathrm{MHz}, \mathrm{HCO}_{2} \mathrm{H}\right) \delta$ : 21.6, $26.2,30.2,54.9,62.5$.

trans-2-Aminocyclohexane-1-sulfonic acid (4h)<smiles>N[C@@H]1CCCC[C@H]1S(=O)(=O)O</smiles>

(

Colorless crystals, m.p. $360{ }^{\circ} \mathrm{C}(\mathrm{dec})$; Lit. ${ }^{12}$ m.p. $410{ }^{\circ} \mathrm{C} .{ }^{13} \mathrm{C}$ NMR $\left(75.5 \mathrm{MHz}, \mathrm{HCO}_{2} \mathrm{H}\right) \delta: 23.3$, $23.4,26.0,30.3,51.3,60.0$.

(S)-Pyrrolidin-2-ylmethanesulfonic acid (4i)<smiles>O=S(=O)(O)C[C@H]1CCCN1</smiles>

Colorless crystals, m.p. $302-5{ }^{\circ} \mathrm{C}(\mathrm{dec})$; Lit. ${ }^{13}$ m.p. $302-4{ }^{\circ} \mathrm{C} .[\alpha]^{20}{ }_{\mathrm{D}}=+32.2\left(c 1.04, \mathrm{H}_{2} \mathrm{O}\right)$; Lit. ${ }^{13}$ $[\alpha]^{20}=+32\left(c\right.$ 3.0, $\left.\mathrm{H}_{2} \mathrm{O}\right) .{ }^{13} \mathrm{C} \mathrm{NMR}\left(75.5 \mathrm{MHz}, \mathrm{HCO}_{2} \mathrm{H}\right) \delta: 21.6,26.2,30.2,54.9,62.5$.

\section{References:}

${ }^{1}$ Xu, J. X. Tetrahedron: Asymmetry 2002, 13, 1129-1134.

${ }^{2}$ Borchardt, D. B.; Bauer, S. H. J. Chem. Phys. 1986, 85, 4980-4988.

${ }^{3}$ Effenberger, F.; Stelzer, U. Tetrahedron: Asymmetry 1995, 6, 283-286.

${ }^{4}$ Baumgarten, H. E.; Fuerholzer, J. F.; Clark, R. D.; Thompson, R. D. J. Am. Chem. Soc. 1963, 85, 3303-3305.

${ }^{5}$ Watson, I. D. G.; Yudin, A. K. J. Org. Chem 2003, 68, 5160 - 5167.

${ }^{6}$ Zhang, Z.; Scheffold, R. Helv. Chim. Acta 1993, 76, 2602-2615.

${ }^{7}$ Shustov, G. V.; Denisenko, S. N.; Chervin, I. I.; Asfandiarov, N. L.; Kostyanovsky, R. G.; Tetrahedron 1985, 41, 5719-5732.

${ }^{8}$ Hassner, A.; Matthews, G. J.; Fowler, F. W. J. Am. Chem. Soc. 1969, 91, 5046-5054.

${ }^{9}$ Grue-Sorensen, G.; White, R. L.; Spenser, I. D. J. Am. Chem. Soc. 1986, 108, 146-158.

${ }^{10}$ Higashiura, K.; Morino, H.; Matsuura, H.; Toyomaki, Y.; Lenaga, K. J. Chem. Soc. Perkin

Trans. 1 1989, 1479-1481.

${ }^{11}$ Braghiroli, D.; Di Bella, M. Tetrahedron Lett. 1996, 37, 7319-7322.

${ }^{12}$ Machetti, F.; Cacciarini, M.; Catrambone, F.; Cordero, F. M.; Romoli, S.; F. De Sarlo, S. J. Chem. Res. (S) 2000, 120-121.

${ }^{13}$ Schwenkkraus, P.; Otto, H.-H. Arch. Pharm. 1990, 323, 93-98. 
Copies of ${ }^{1} \mathrm{H}$ and ${ }^{13} \mathrm{C}$ NMR Spectra of the Acetamides and N-Acetyl Substituted Taurines<smiles>CC(=O)NC(CS)Cc1ccccc1</smiles>

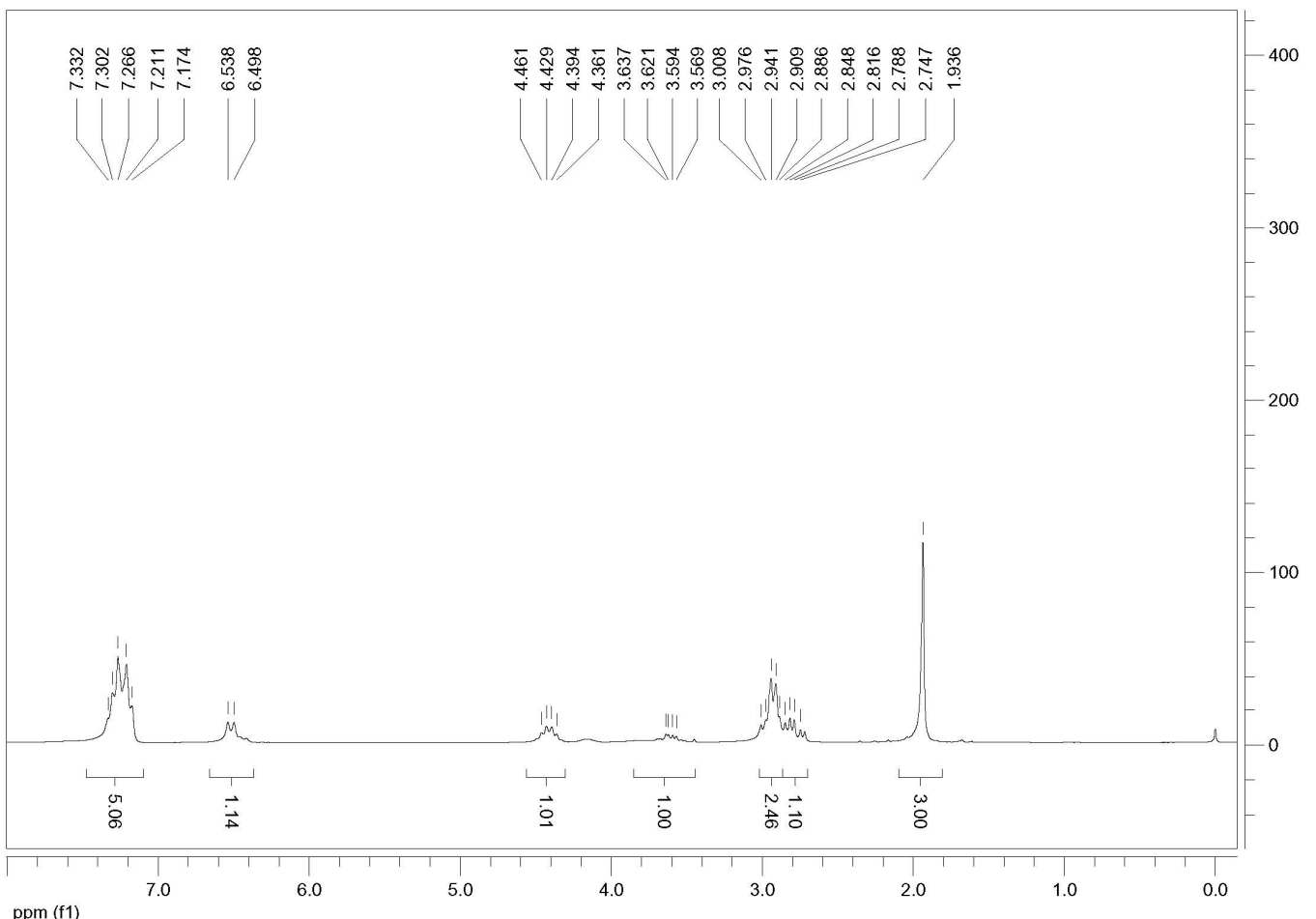

ppm (f1)<smiles>CC(=O)NC(CS)Cc1ccccc1</smiles>

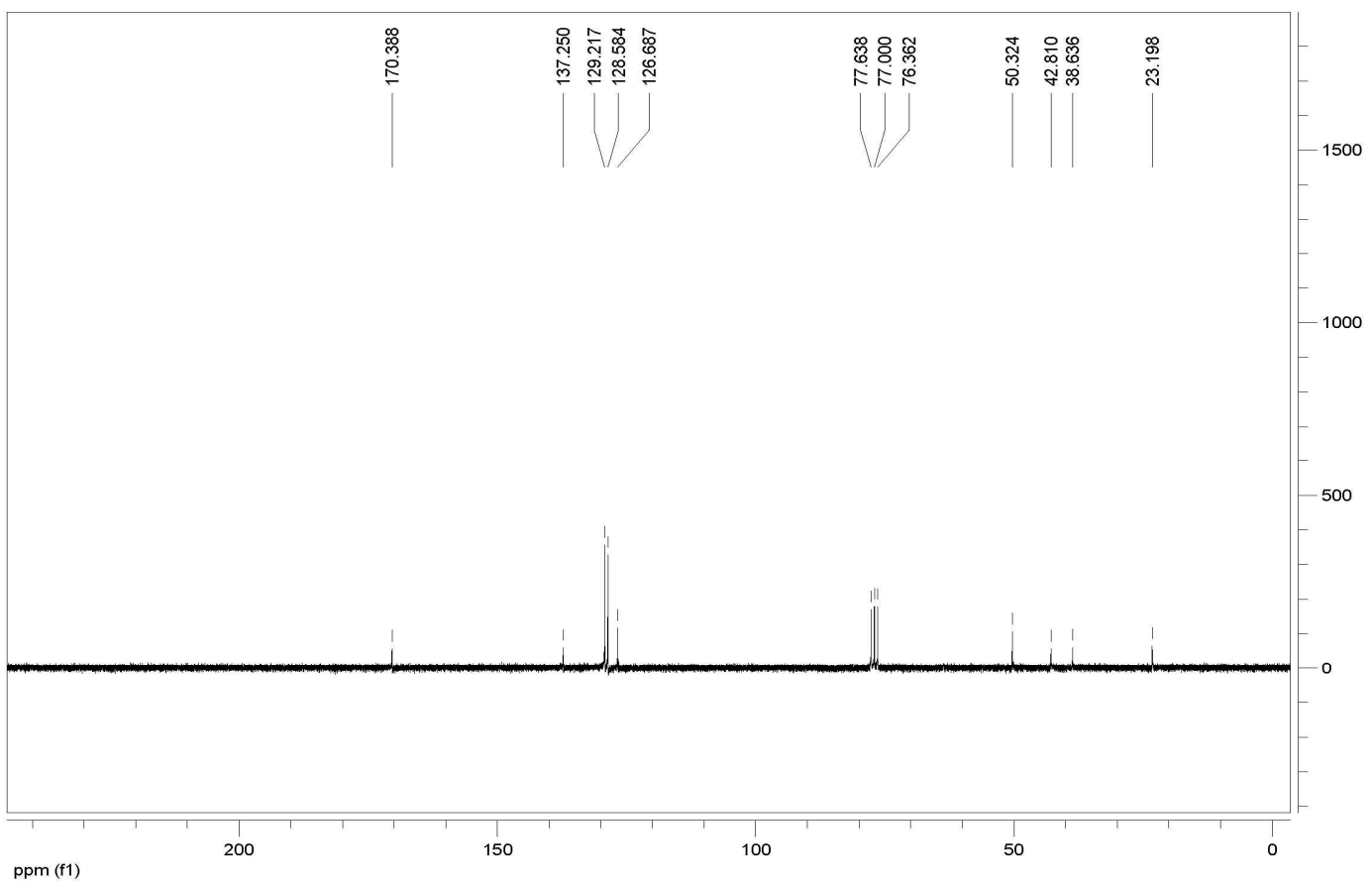




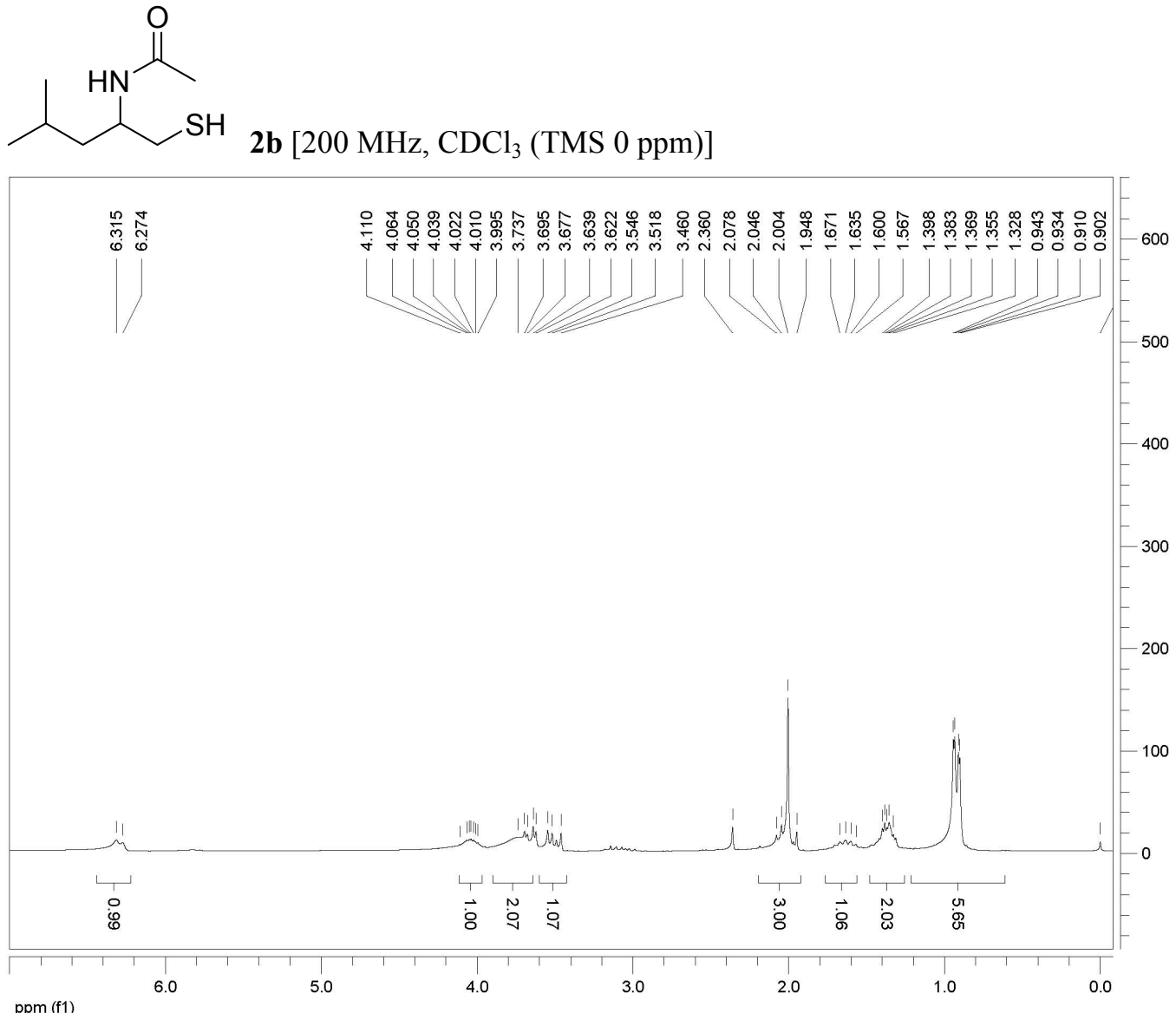

ppm (f1)<smiles>CC(=O)NC(CS)CC(C)C</smiles>

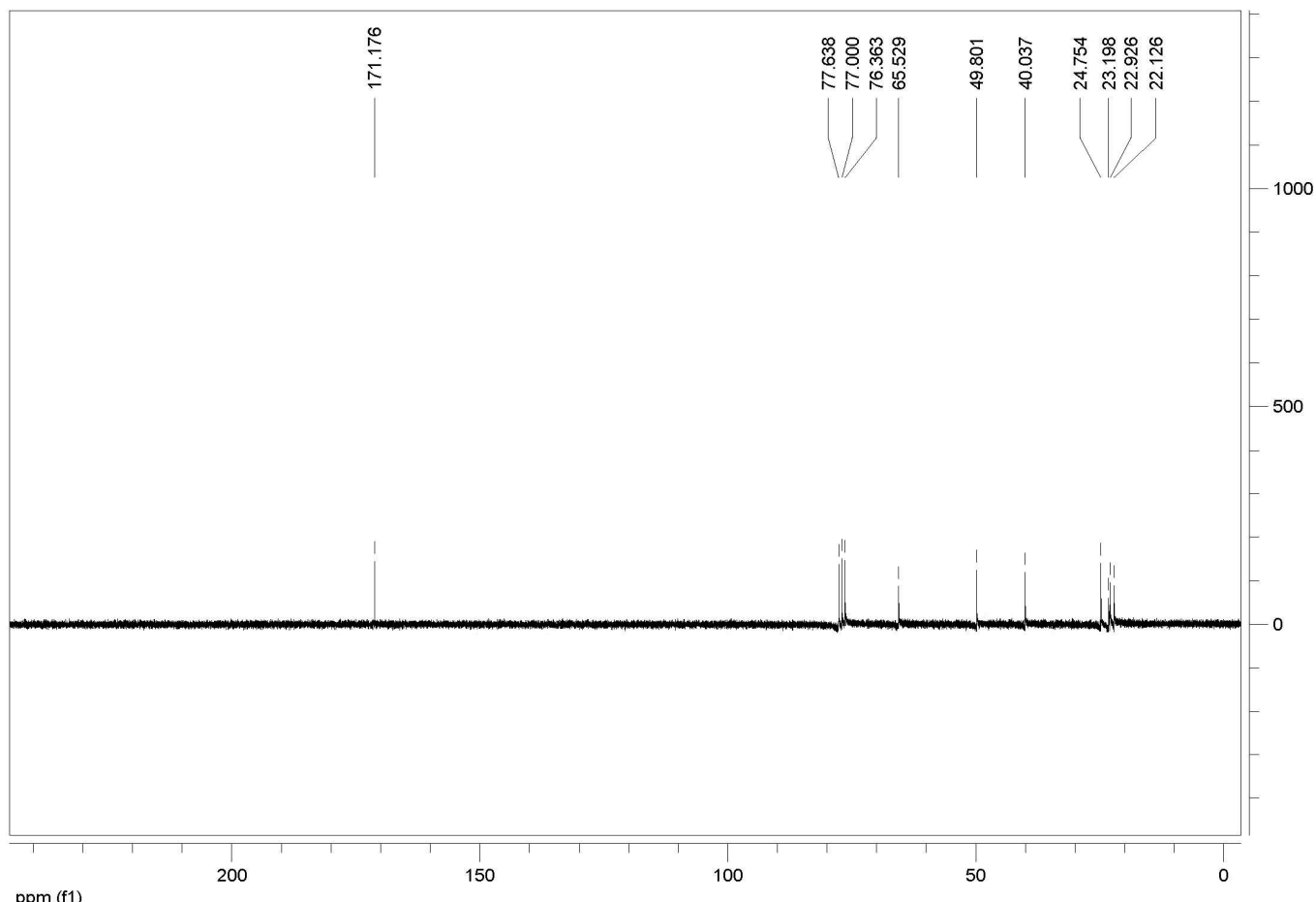

ppm (f1) 
$\mathrm{SO}_{3} \mathrm{H}_{\mathbf{3 a}\left[200 \mathrm{MHz}, \mathrm{D}_{2} \mathrm{O}(4.67 \mathrm{ppm})\right]}$

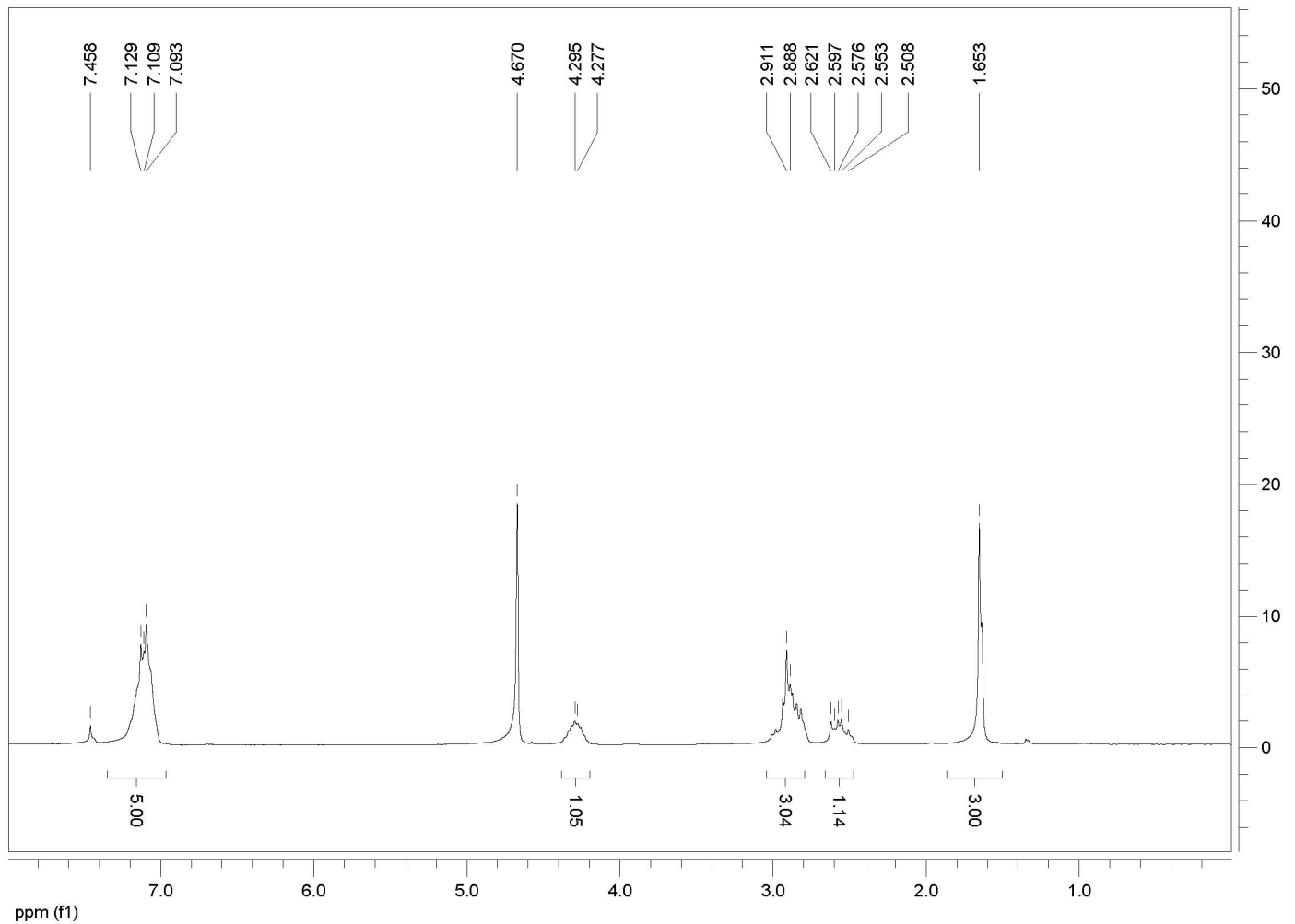

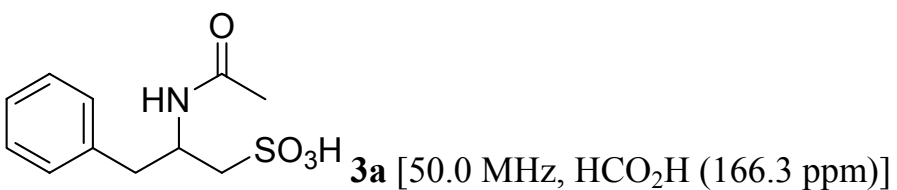

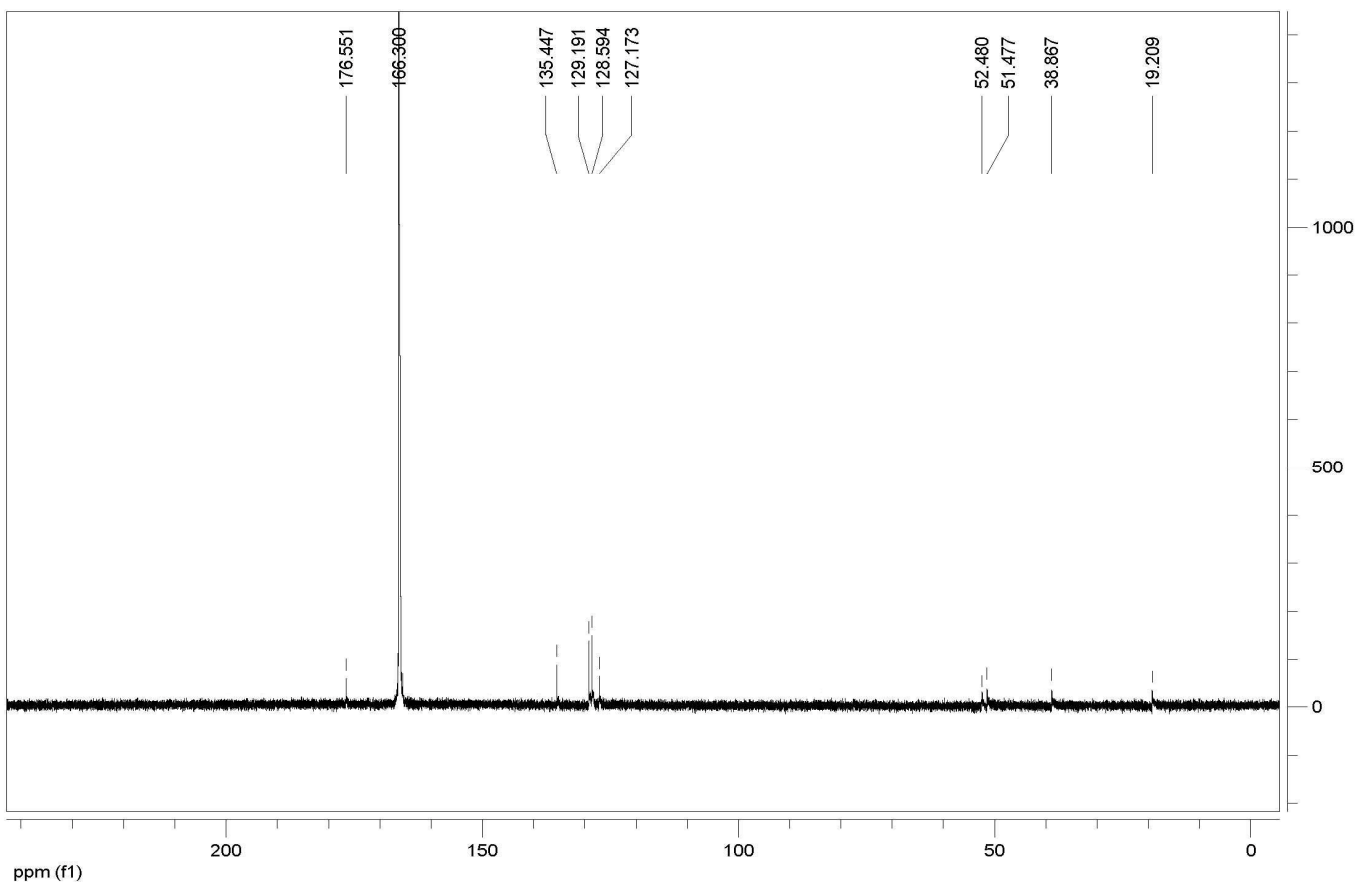




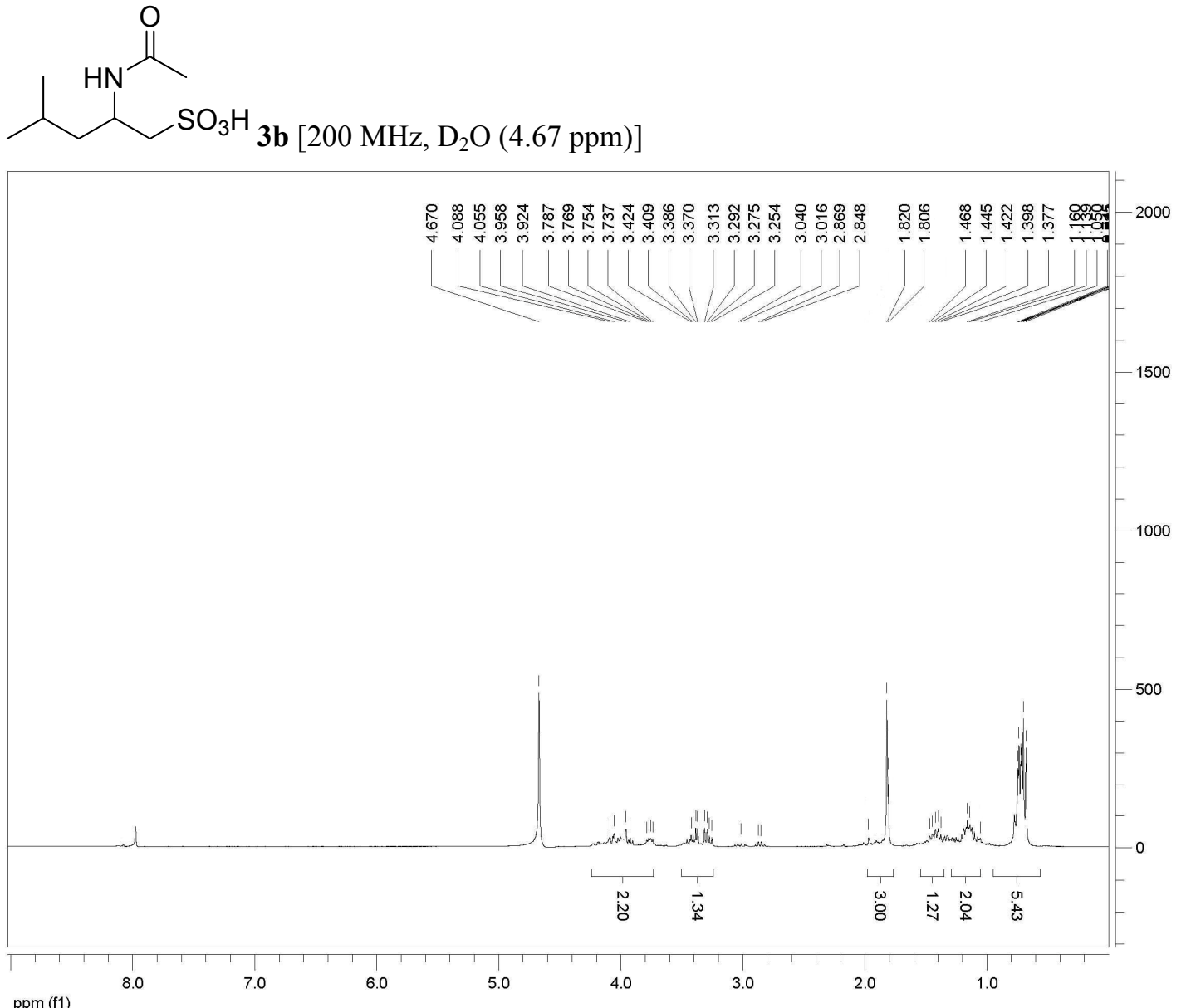

ppm (f1)

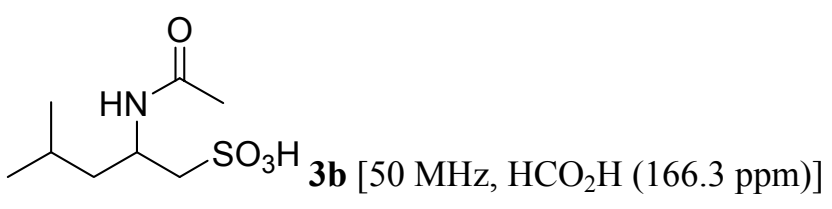

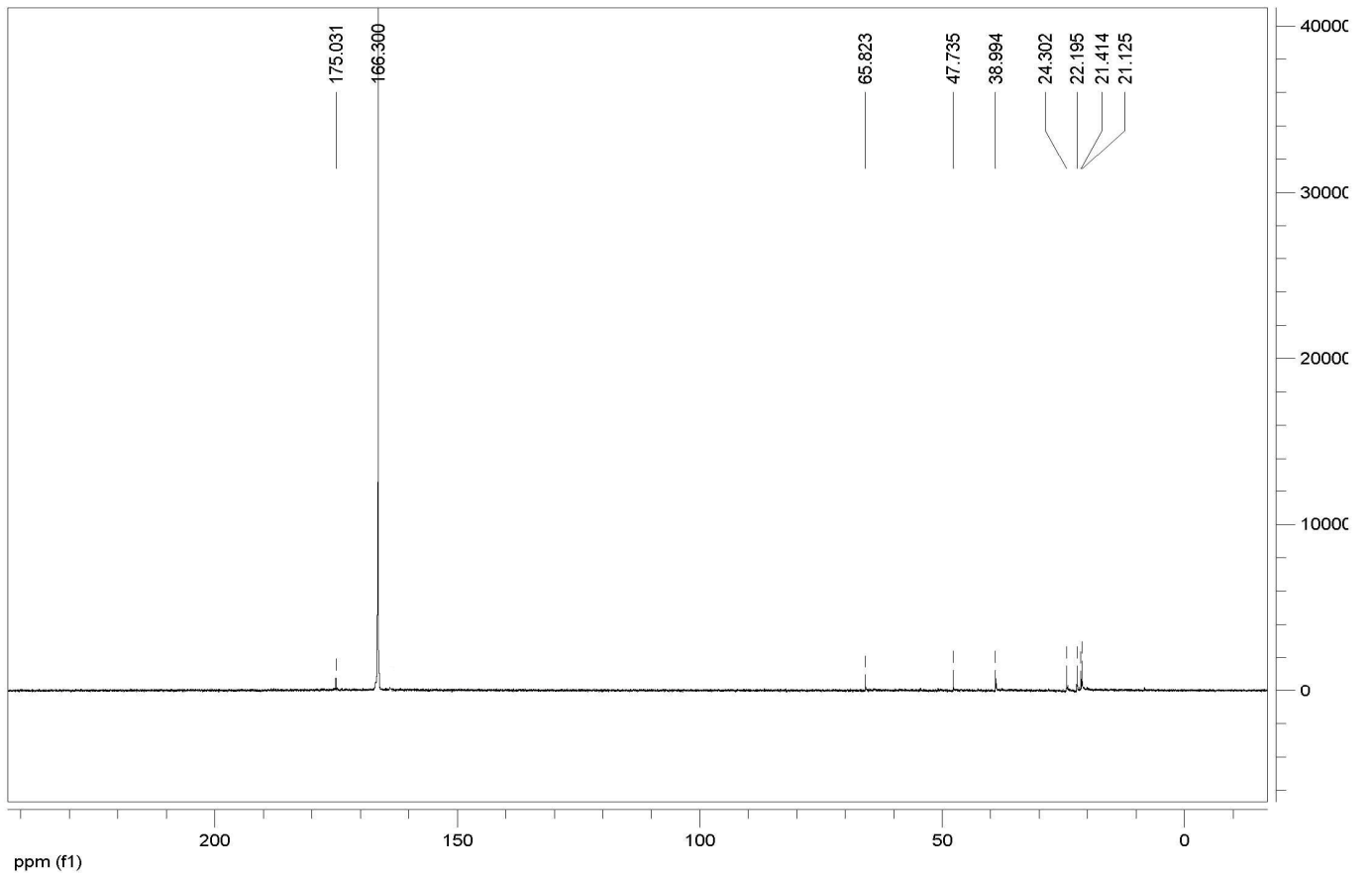


Copies of ${ }^{13} \mathrm{C}$ NMR Spectra of the Known Substituted Taurines and ${ }^{1} \mathrm{H}$ and ${ }^{13} \mathrm{C}$ NMR Spectra of Taurine and the Unknown Substituted Taurines

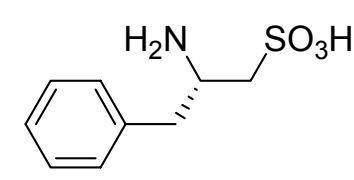

4a $\left[75.5 \mathrm{MHz}, \mathrm{HCO}_{2} \mathrm{H}(166.3 \mathrm{ppm})\right]$
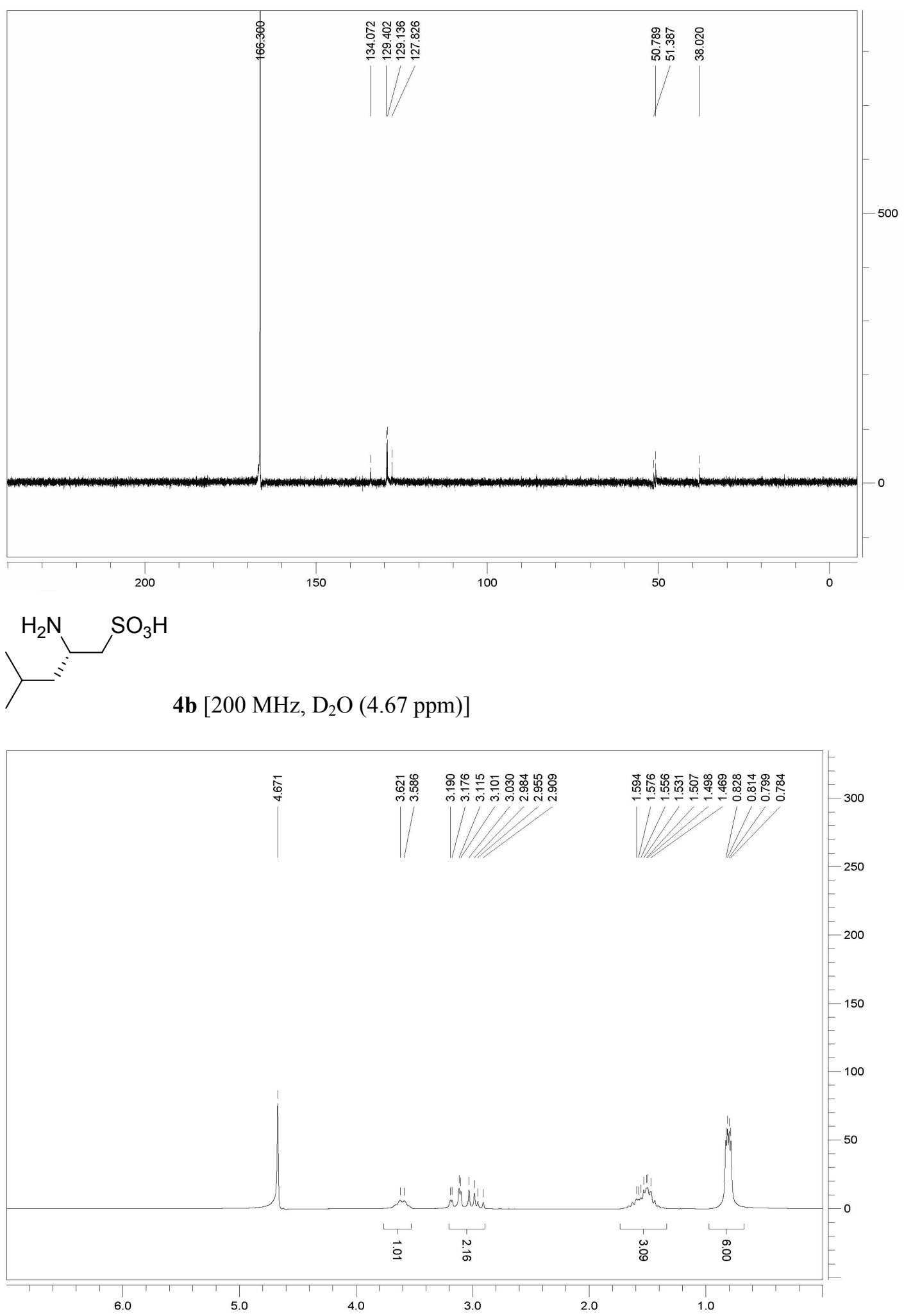


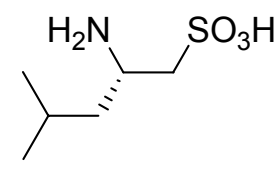

4b [75.5 MHz, $\left.\mathrm{HCO}_{2} \mathrm{H}(166.3 \mathrm{ppm})\right]$

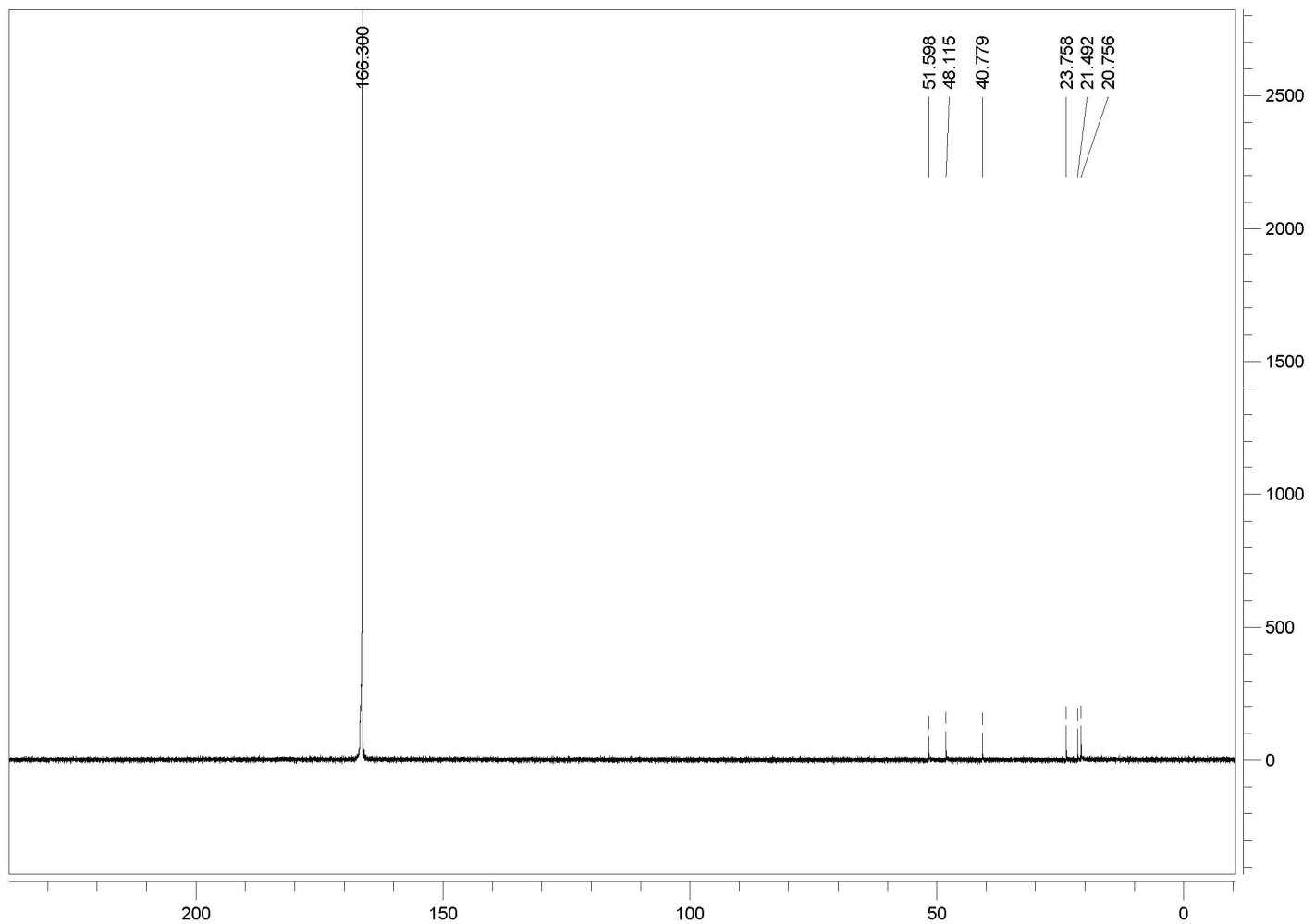

$\mathrm{H}_{2} \mathrm{~N}$

$\mathrm{SO}_{3} \mathrm{H}_{4 \mathbf{c}}\left[200 \mathrm{MHz}, \mathrm{D}_{2} \mathrm{O}\right.$ (4.67 ppm)]

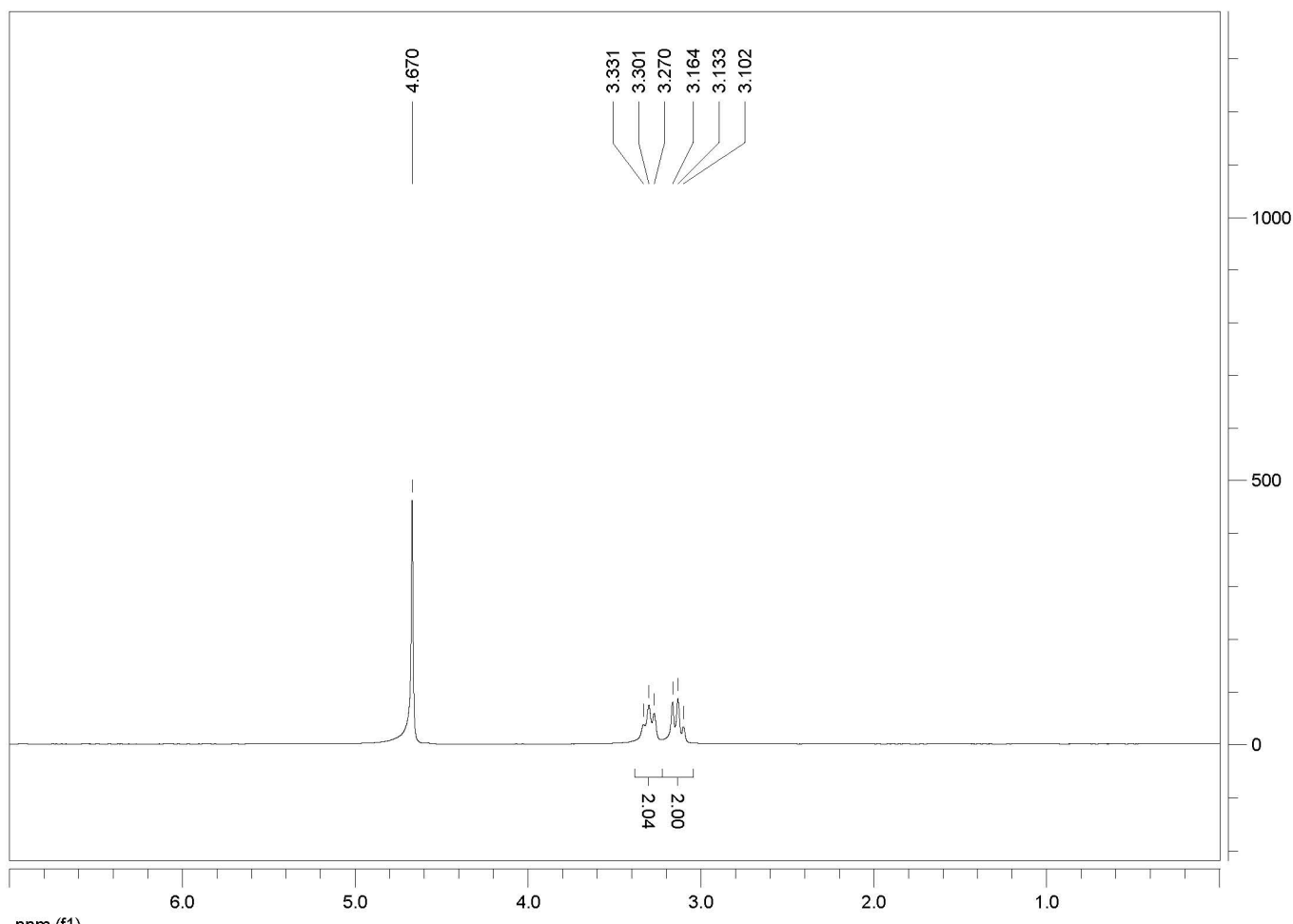

ppm (f1) 
$\mathrm{H}_{2} \mathrm{~N}$

$\mathrm{SO}_{3} \mathrm{H}_{4 \mathbf{c}}\left[50.0 \mathrm{MHz}, \mathrm{HCO}_{2} \mathrm{H}(166.3 \mathrm{ppm})\right]$

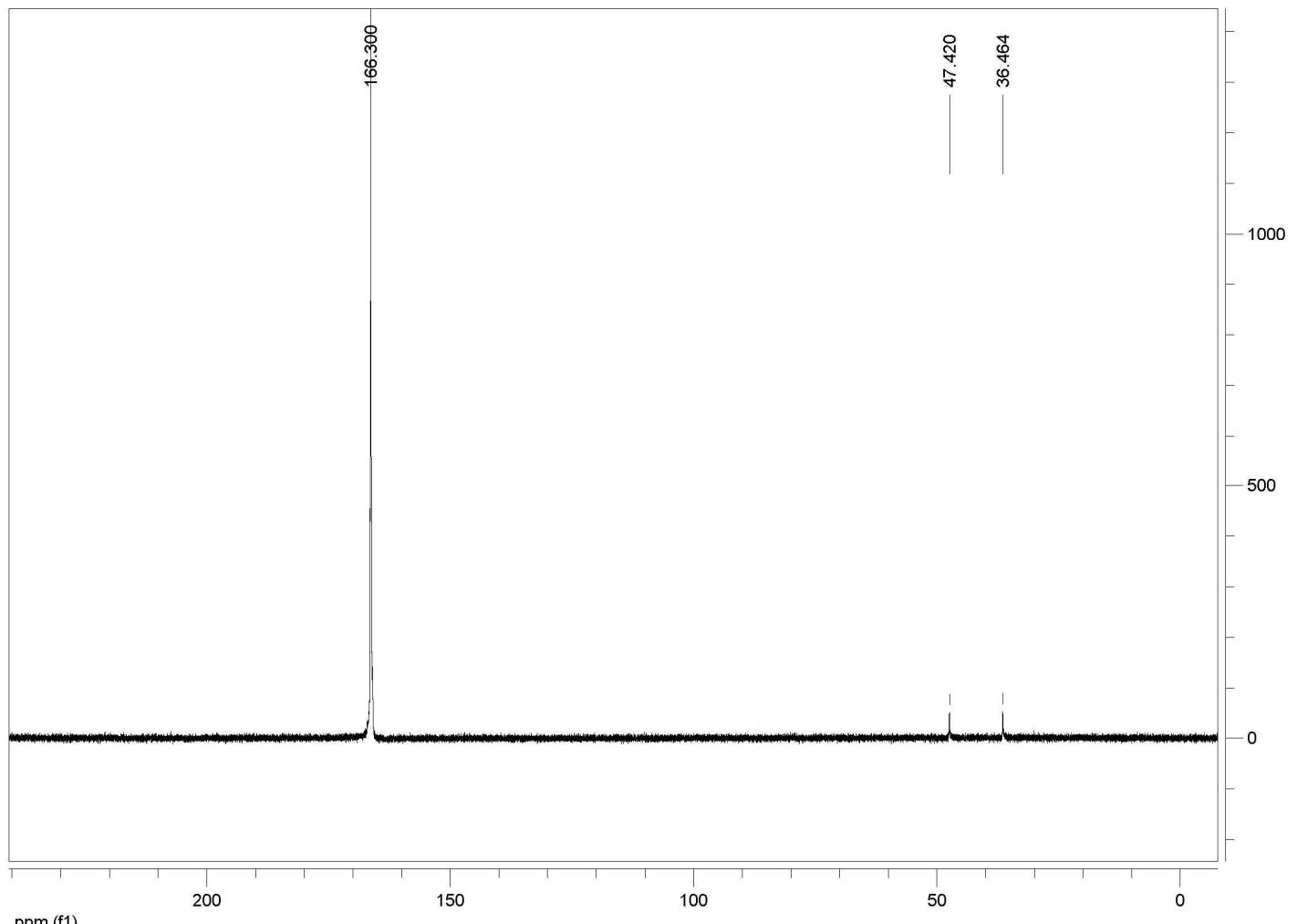

ppm (f1)

$\mathrm{H}_{2} \mathrm{~N} \quad \mathrm{SO}_{3} \mathrm{H}$

4d $\left[75.5 \mathrm{MHz}, \mathrm{HCO}_{2} \mathrm{H}(166.3\right.$ ppm)]

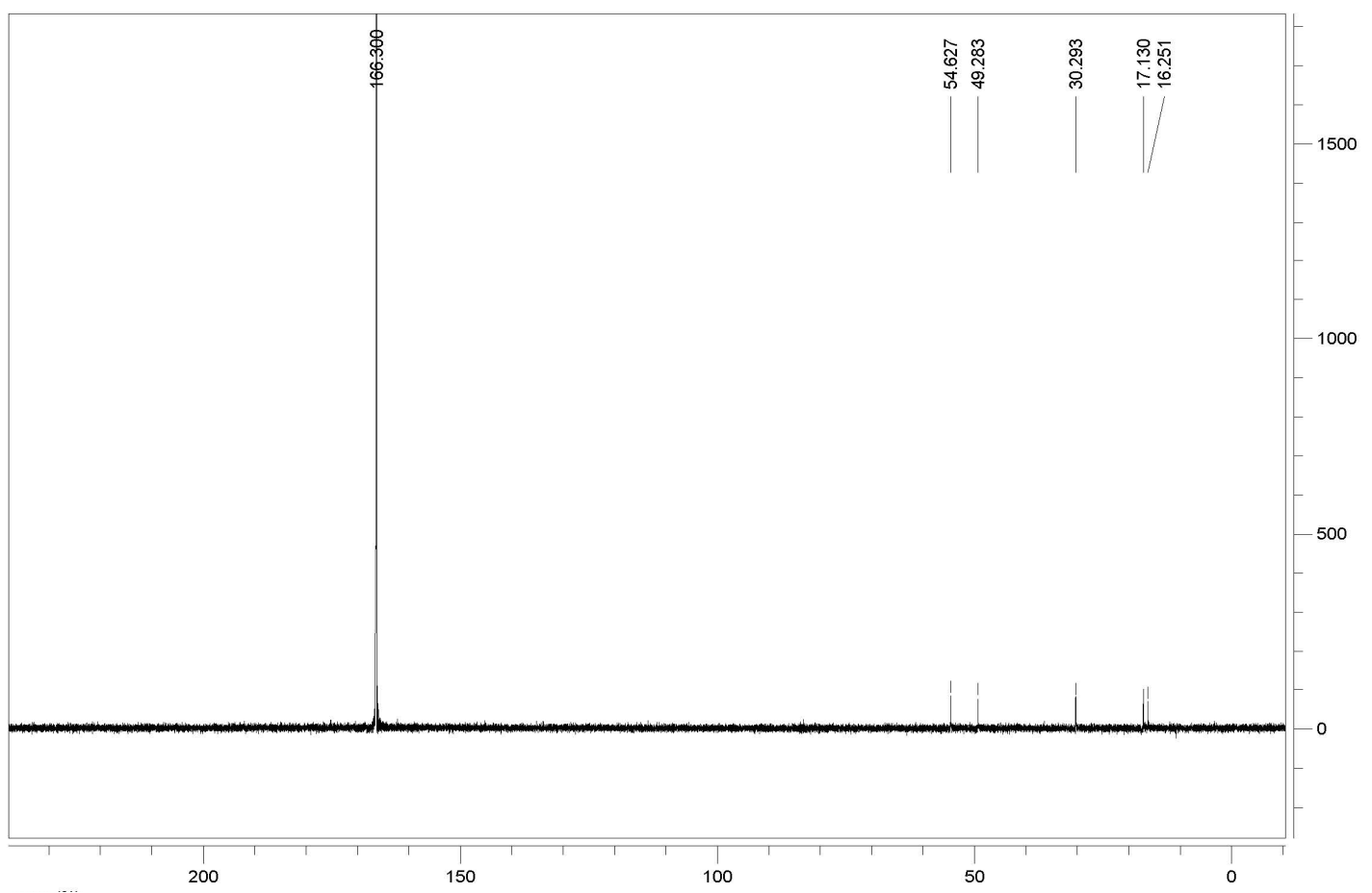




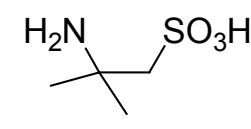

4e [75.5 MHz, $\left.\mathrm{HCO}_{2} \mathrm{H}(166.3 \mathrm{ppm})\right]$

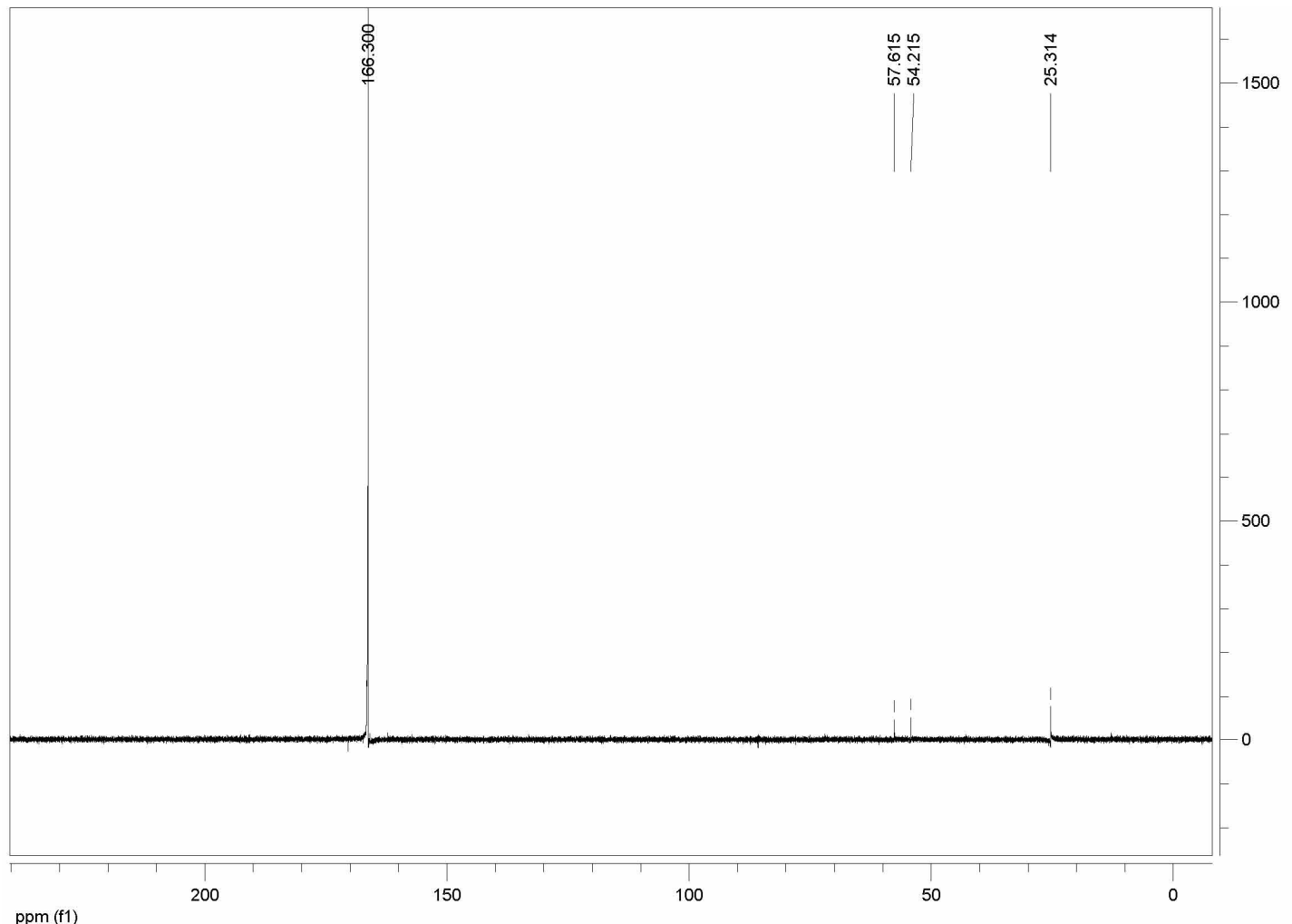

ppm (f1)
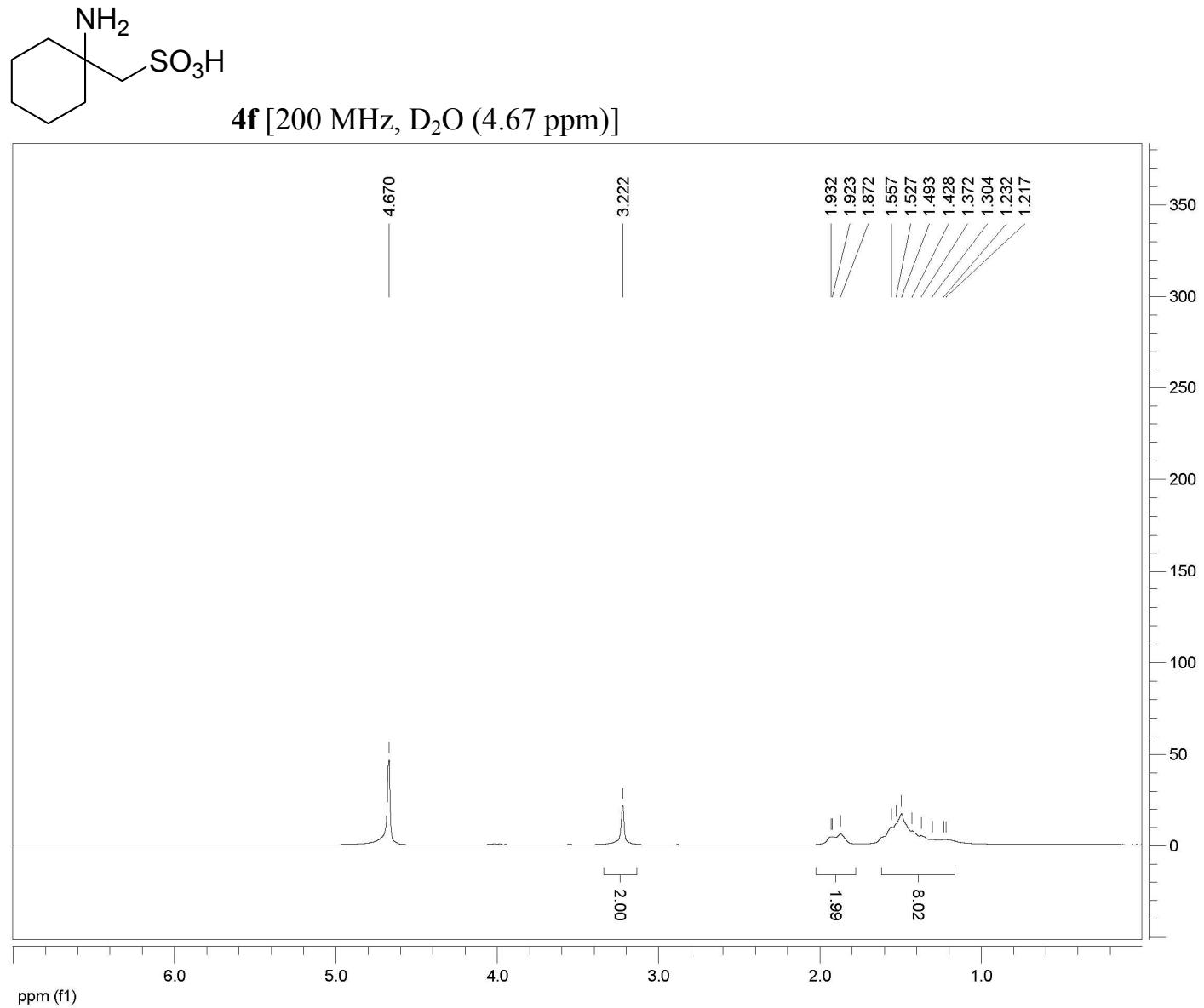


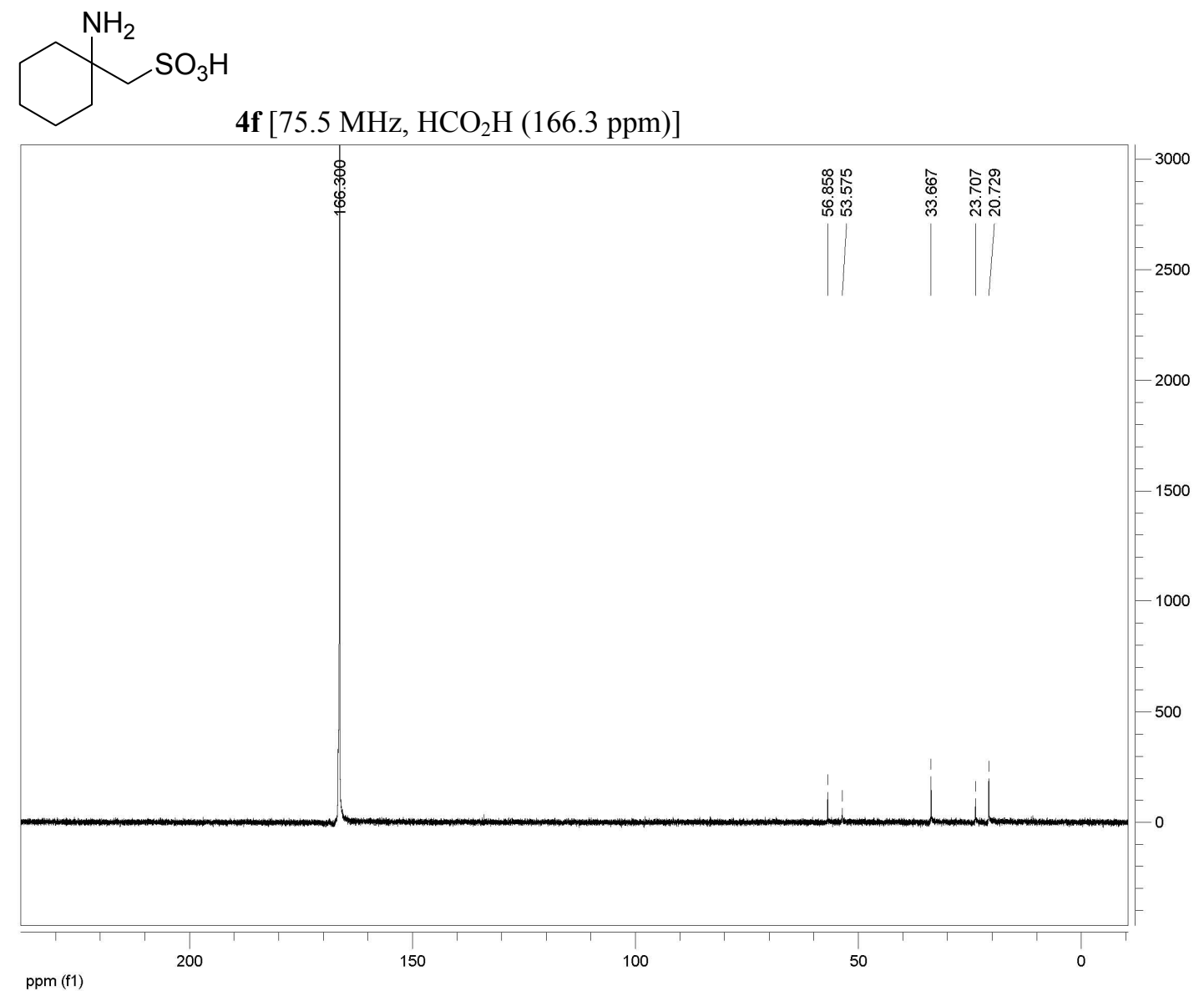

$\square^{{ }^{\prime} \mathrm{SO}_{3} \mathrm{H}}$

(士) $\quad 4 \mathbf{g}\left[75.5 \mathrm{MHz}, \mathrm{HCO}_{2} \mathrm{H}(166.3 \mathrm{ppm})\right]$

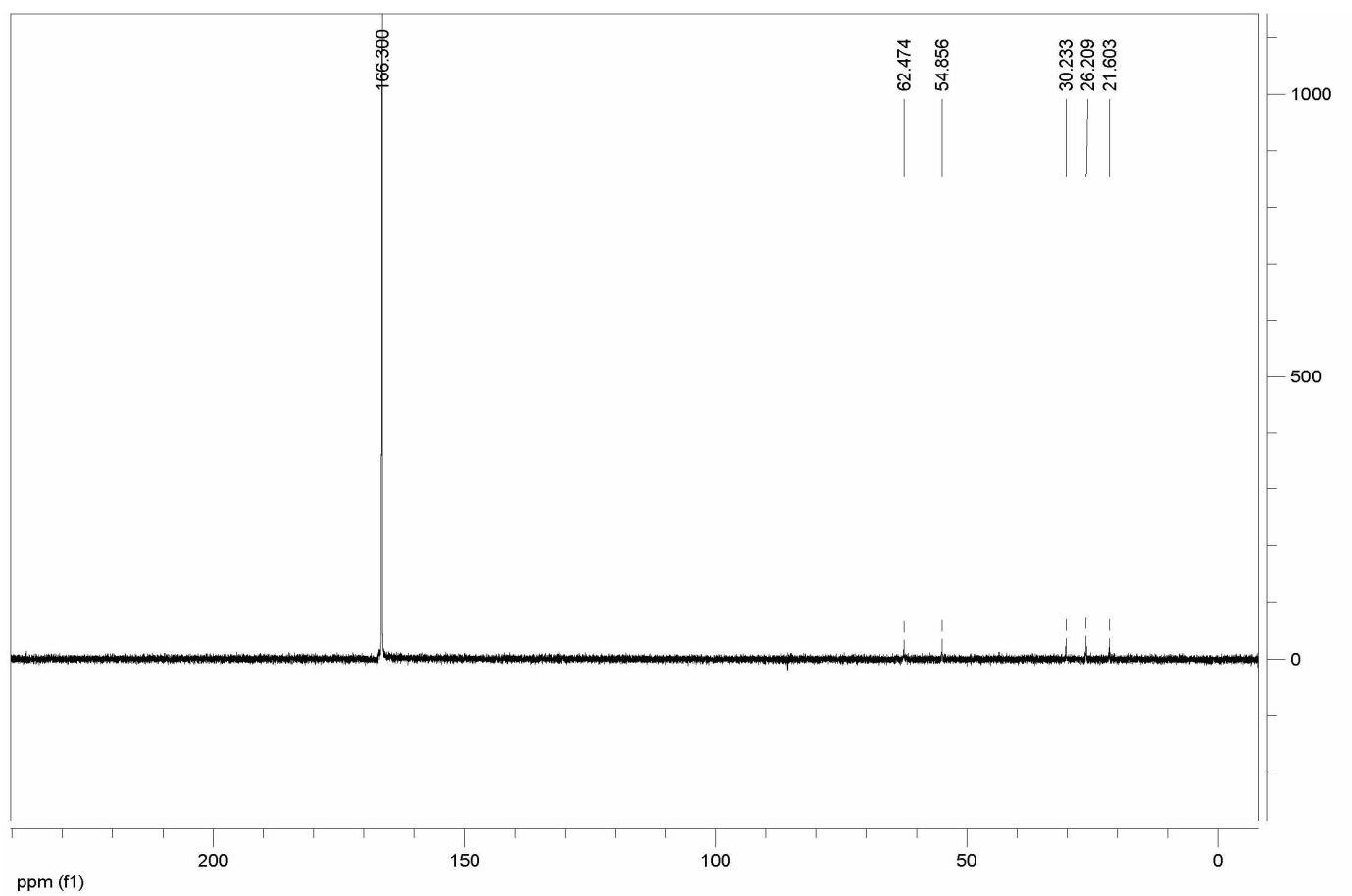




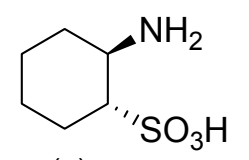

$( \pm)$

4h [75.5 MHz, $\left.\mathrm{HCO}_{2} \mathrm{H}(166.3 \mathrm{ppm})\right]$

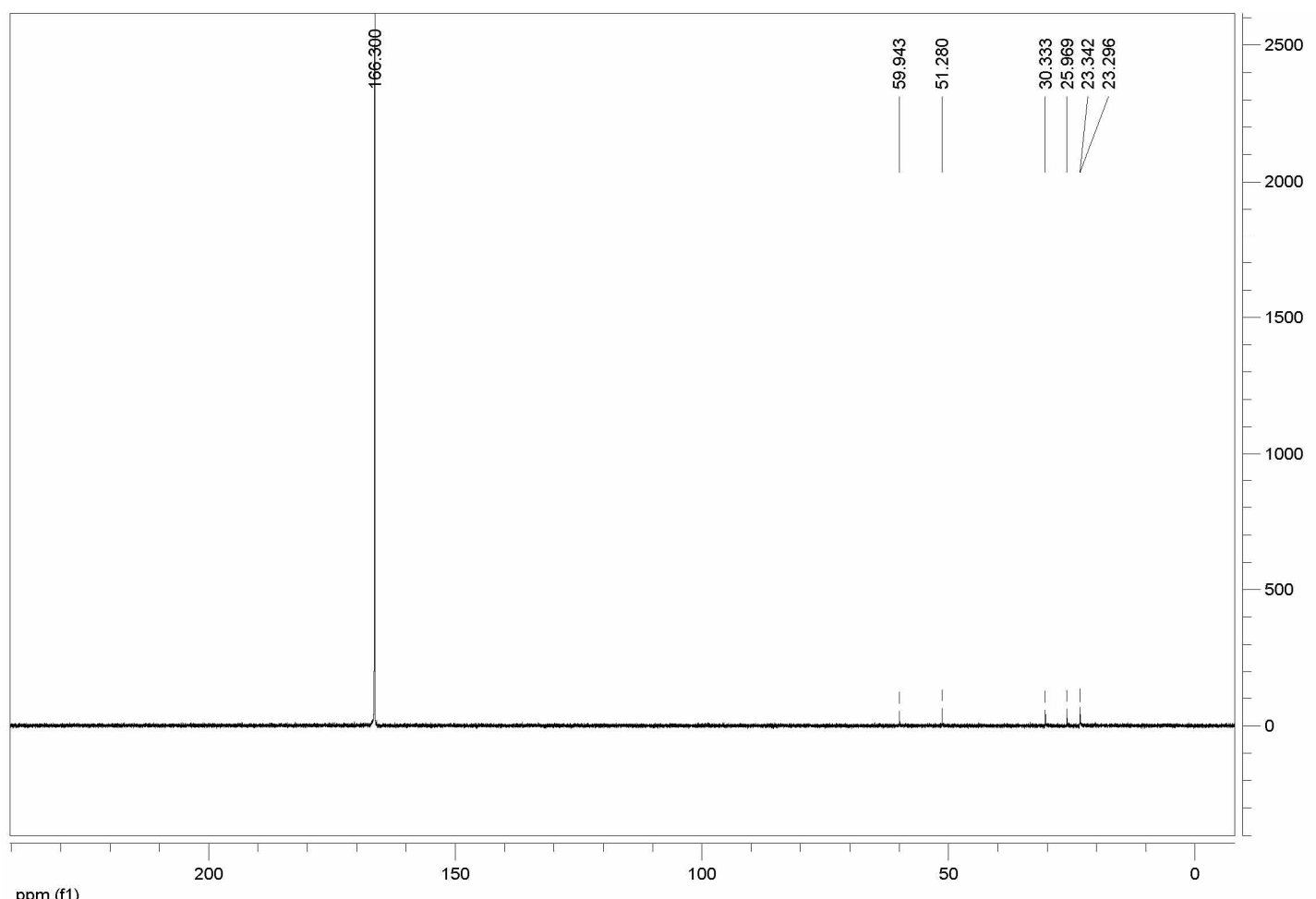

S $\mathrm{SO}_{3} \mathrm{H}$

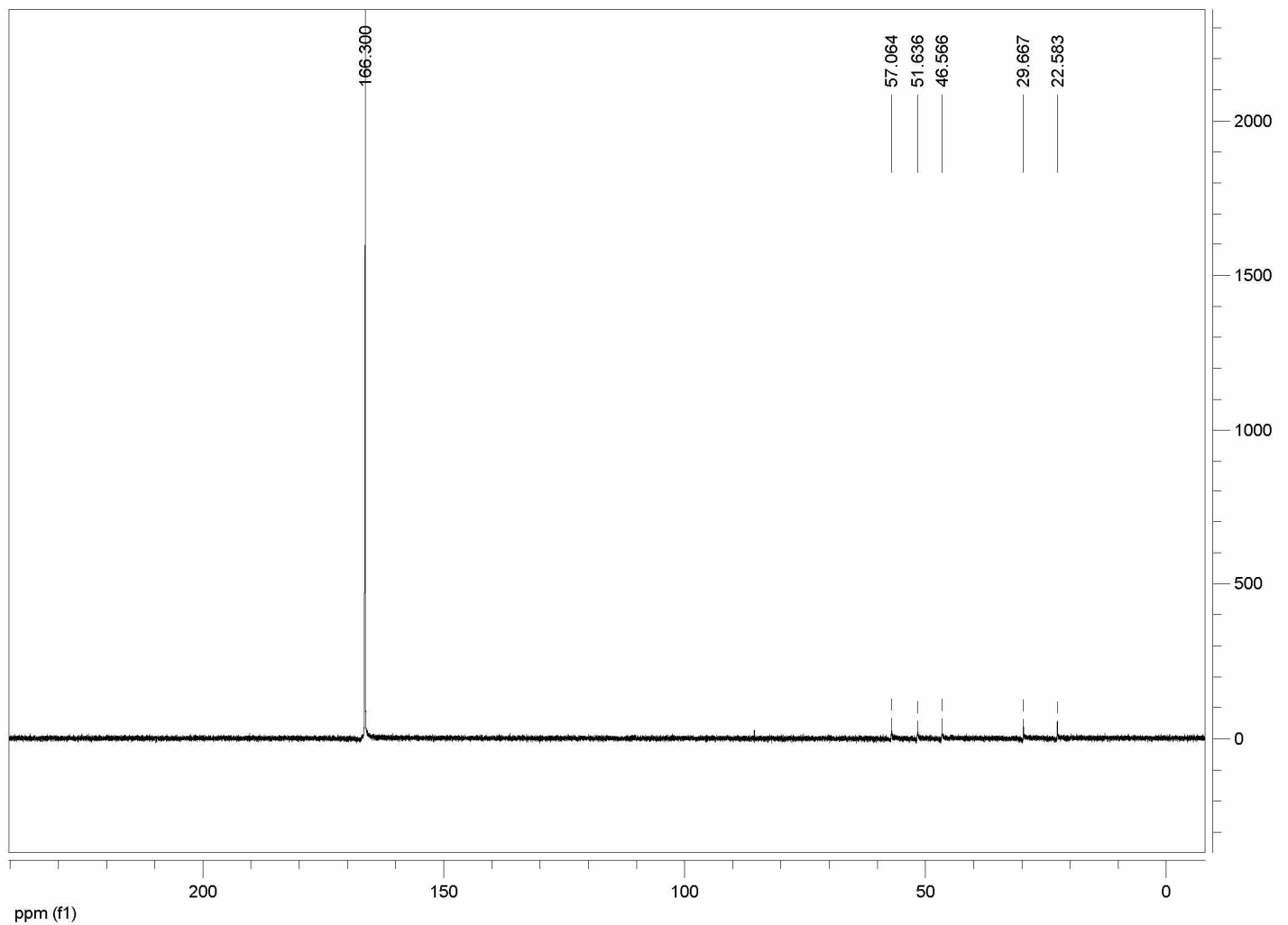

